\title{
Spreading of Human Babesiosis in China: Current Epidemiological Status and Future Challenges
}

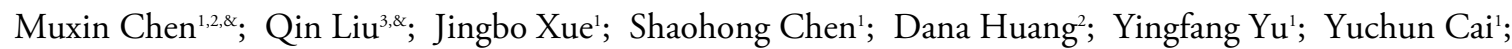 \\ Yan $\mathrm{Lu}^{1}$; Peng Song'; Renli Zhang ${ }^{2}$; Lin Ai ${ }^{3, * ;}$; Jiaxu Chen ${ }^{1, *}$
}

Human babesiosis is an infection caused by intraerythrocytic protozoa of the genus Babesia and is a worldwide emerging tick-borne disease (1). Babesia is mainly parasitized in erythrocytes of human or other vertebrates and transmitted by the bite of ticks, blood transfusion, or organ transplantation (1). Humans are generally susceptible to Babesia, especially those who are immunocompromised with conditions such as splenectomy or splenic dysfunction, as well as those of old age, with infirmity, or with other severe diseases.

The life cycle of Babesia includes a definitive host and an intermediate host, and its definitive host (vector) is Ixodes (2). The main species Ixodes that can transmit Babesia include Dermacentor nuttalli, D. silvarum, D. niveus, $D$. sinicus, Rhipicephalus haemaphysaloides, Haemaphysalis longicornis, Ixodes persulcatus, and I. ricinus. Babesia species have a wide range of intermediate hosts including humans, rodents (such as Niviventer niviventer, Rattus norvegicus, Rattus flavipectus, and Apodemus agrarius), and other animals (e.g., cattle, deer, dogs, raccoons, and birds). Since Babesia bigemina was first discovered in red blood cells of infected cattle in 1888 by Babes (3), more than 100 species of Babesia have been identified and reported. However, only B. microti, B. diversens, $B$. duncani, $B$. venatorum, and other species can infect humans (2).

\section{CURRENT EPIDEMIOLOGICAL STATUS OF BABESIOSIS OUTSIDE OF CHINA}

Babesia is distributed worldwide. After the first case of human babesiosis was reported by Yugoslavia in 1957 (4), multiple human cases of babesiosis have been reported in Asia, Africa, and Oceania, and significant numbers have been reported in American and European countries. The United States has reported the most human cases of babesiosis worldwide, and the primary species include $B$. microti, $B$. duncani, and $B$. divergens-likes organisms (5). According to data released by the Centers for Disease Control and Prevention (Atlanta, GA, USA) in 2019, there were
7,612 cases of babesiosis (6,277 confirmed [82.5\%] and 1,335 suspected [17.5\%]) in the United States from 2011 to 2015, which occurred most frequently from June to August in the Northeast and Upper Midwest of the US, and B. microti was the most common Babesia species (G). Moreover, there were three main species of Babesia recorded in Europe: $B$. diversens, $B$. ventorum, and $B$. microti $(5,7)$. The first case of babesiosis was reported in France in 1976 (8). In 1979 , the first case of $B$. diversens was diagnosed in Scotland, and the patient was a 34-year-old male who underwent splenectomy (9). I. ricinus was the main vector of human Babesia infection in Europe. In addition, human cases of babesiosis have also been reported in several countries (e.g., Egypt, Mexico, South Africa, Mozambique, Australia, Brazil, Japan, Republic of Korea, Singapore, United Kingdom, Russia, Spain, etc.) $(1,5,10-11)$.

\section{CURRENT EPIDEMIOLOGICAL STATUS OF BABESIOSIS IN CHINA}

The first reported case of human Babesia infection in China dates back to 1944 . Hong Shilu examined the blood smear of a suspected malaria patient in Chongqing and demonstrated that the patient was infected with Babesia according to an analysis of the morphological characteristics of the protozoa (12). From 1944 to 2019, more than 314 cases of human babesiosis or asymptomatic infection have been reported in China, which were distributed in 14 provincial-level administrative divisions (PLADs) (Figure 1). The greatest number of human babesiosis cases was reported in Heilongiiang Province in the northeast of China including 58 cases of $B$. crassa-like infection, 48 cases of $B$. venatorum infection, 13 cases of $B$. microti asymptomatic infection, and 1 case of Babesia infection without an identified species (13-15). More than 20 cases of Babesia infection were reported in Chongqing, Yunnan, and other PLADs located in Southwest China. Of these, there were 10 


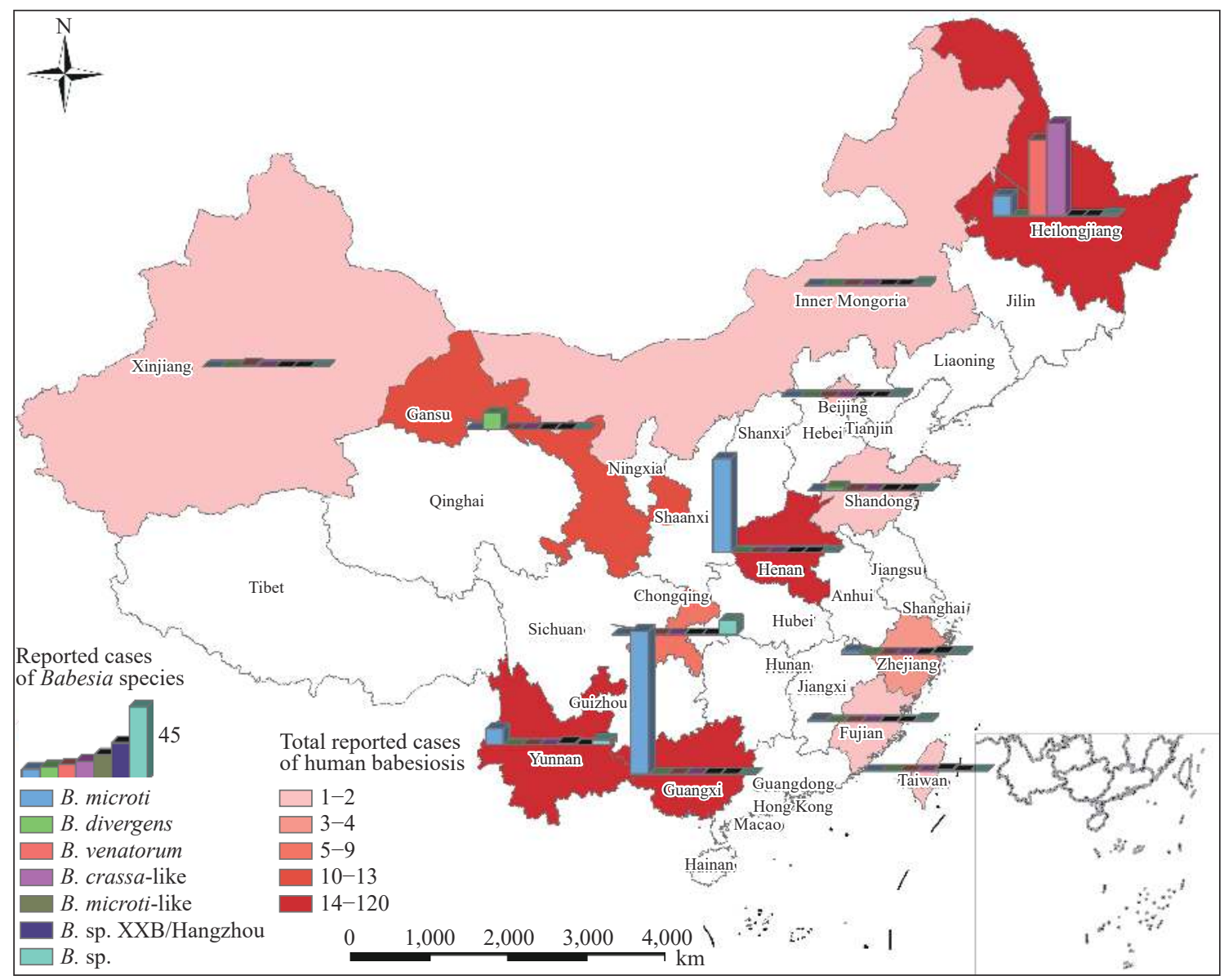

FIGURE 1. Distribution of human babesiosis in China from 1944 to 2019 . Abbreviations: $B .=B a b e s i a$; sp.=species.

cases of the co-infection of Babesia and Plasmodium in China-Myanmar border areas. A total of six cases of babesiosis were reported in East China, and several Babesia species were identified in this area including $B$. diversens, B. microti, etc. (15-20). The main species distributed across China included $B$. microti, $B$. venatorum, $B$. diversens, and $B$. crassa-like infections. In southern China, $B$. microti was the main species, while in nothern China, $B$. venatorum and $B$. crassa-like were the main species with the main vectors as $H$. longicornis, $R$. haemaphysialoids, I. persulcatus, etc. Human babesiosis have occurred most frequently in summer, as well as in areas where the tick vector, vertebrate reservoirs, and deer were near human $(15,19)$. An estimated $98.41 \%(309 / 314)$ of human babesiosis cases were reported in the past 10 years. Among the 314 cases, cases have been reported with a history of tick bites (93 cases, 29.62\%), blood transfusion (1 cases, $0.32 \%$ ), and had a splenectomy (2 cases, $0.64 \%$ ), and some patients reported no clear route of infection (221 cases, $70.38 \%)$ in China (15-18).

\section{CLINICAL MANIFESTATIONS}

The clinical manifestations of human babesiosis are quite different and are closely associated with the Babesia species and the immune system function of the infectee with an incubation period of 1 to 4 weeks. Patients with normal immune function had selflimiting infections, and symptoms lasted for 2 to 4 weeks. Common clinical manifestations include chills, fever, sweating, fatigue, nausea, anorexia, muscle pain, joint pain, headache, abdominal pain, anemia, and other symptoms similar to influenza. Patients with severe babesiosis usually have an acute onset, and most experienced age-related muscle weakness and immunodeficiency. Severe clinical manifestations include high fever, severe anemia, hemoglobinuria, jaundice, respiratory distress, renal failure, coma, etc. Sometimes, body temperatures reach $40{ }^{\circ} \mathrm{C}$ and can be confused with symptoms of malaria $(1,15)$. Laboratory blood smear staining with Giemsa stain is the most applied test method. However, due to its variable morphology, there are many stages (sporozoite, trophozoite, merozoite, gametocyte, etc.) of the 
protozoa on the same blood smear, and the trophozoite of Babesia can easily be misdiagnosed as Plasmodium falciparum in Babesia and Plasmodium mixed endemic area and needs to be differentiated from Plasmodium falciparum with the aid of immunological assays, molecular biology tests, and other auxiliary laboratory methods (19).

To date, babesiosis has not significantly attracted the attention of Chinese clinicians. Although there are diagnostic criteria related to clinical manifestations, laboratory etiology, immunology, and molecular biological testing of human babesiosis, babesiosis maintains relatively low awareness and is often neglected by practitioners, which causes great difficulties for the diagnosis of the disease by Chinese medical staff and results in increased missed diagnoses and misdiagnoses. It has increased the disease's burden on patients and detrimental effects on people's health.

\section{CHALLENGES}

With the changes due to global warming on the ecological environment, economic globalization, the increasing frequency of international communication, the rapid growth of the population, enhanced interactions between human and wildlife populations, etc., the risk of humans contracting tick-borne and other vector-borne diseases has been greatly increased. Since the symptoms of human babesiosis resemble that of malaria and diagnosis is predominantly reliant on microscopic evaluation of blood smears, this disease may often be misdiagnosed as a $P$. falciparum infection, especially for imported cases from areas with Babesia and Plasmodium co-endemicity. Performing early diagnosis and treatment requires more research attention. For humans, it was of great significance to use antimalarials (e.g., atovaquone with azithromycin, quinine plus clindamycin) for Babesia parasites, while highly immunocompromised individuals respond poorly to those medications. Although Babesia and its case characteristics have been described earlier by Chinese scientists, previous studies on Babesia focused on the field of veterinary medicine, and human population babesiosis screening and related scientific research has been insufficient. People with lowered immune system functioning are susceptible to Babesia, so the prevalence of babesiosis among HIV-infected people should also be monitored.

Babesiosis, as an emerging parasitic disease, has gradually attracted immunologists' attention worldwide (21). In the past 10 years, Chinese scientists have done a lot of research on Babesia, including detection (morphological test, immunological test, and molecular biology test, etc.), monitoring (case surveillance of human babesiosis, the surveillance of the animal host and vector tick), vector tick control, the establishment of vector risk early warning model, etc. After doing several years of research on prevention and control of human babesiosis in China, there are still great challenges. 1) Misdiagnosis: due to the similarity in morphology, pathogenicity and clinical symptoms to those of malaria, this disease is easy to be misdiagnosed. After elimination of malaria, the prevalence of babesiosis may cause inexperienced clinicians to be misdiagnosed as malaria cases, thus affecting accurate judgment of malaria elimination results in China. 2) Medical attention: the majority of clinicians have no awareness of babesiosis, and mentioning the disease is neither rational nor perceptual, resulting in the increase of missed diagnosis and misdiagnosis. 3) Intermediated host: the increasing of tick bites caused by the increasing frequency of international exchanges, cross-border travel, the elevation of interactions between people and wild animals, and prominent problems of travel medicine including the increased opportunity for exposure and risk for imported babesiosis. 4) Public awareness: the public's awareness about babesiosis is insufficient and leads to inadequate protection of field activities and increased opportunities for infection. 5) Detection: lack of screening for Babesia and other pathogens in blood products in China is a continued problem that emerged several decades ago. 6) Research funding: due to lack of government funding, few basic studies on Babesia in China have been conducted, and prevention and control strategies cannot be continuously and effectively carried out.

\section{DIAGNOSTIC PROSPECTS}

In this regard, doctors affiliated to medical departments, hospitals, blood centers, and disease prevention and control institutions need to pay further attention to this neglected tropical disease. First, strengthening the training of health researchers to include the morphology, pathogenicity, clinical symptoms, and diagnosis of babesiosis and malaria will better consolidate the major achievements of malaria elimination in China. Second, it is necessary to enhance the publicity and education of prevention and control of babesiosis for tourists and potential risk groups in babesiosis endemic area and to elevate the monitoring of returning tourists and key groups of fever and immunocompromised patients (e.g., HIV 
infections, with splenectomy or splenic dysfunction, etc.) in babesiosis endemic areas by reporting suspected cases to China CDC immediately. Third, the screening of Babesia in blood products must be strengthened to ensure the safety of blood products in order to avoid causing unnecessary biosafety events. Fourth, further cooperation must be called for related to health, agriculture, and environmental protection to effectively develop basic research and prevention and control strategies for neglected tropical diseases (e.g., babesiosis), in order to ensure the health of Chinese people and make contributions to the prevention and control concept of China's goal of "One Health".

Fundings: This study was financially supported by the General Program Shanghai Municipal Commission of Health and Family Planning of China (Grant No. 201840286 and 201640278), the Program for the Shanghai Natural Science Foundation of China (Grant No. 18ZR1443500), the Youth Science Foundation of Chinese Center for Disease Control and Prevention (Grant No. 2018A105), the Shenzhen San-Ming Project for prevention and research on vector-borne diseases (Grant No. SZSM201611064), the National Science and Technology Major Program of China (Grant No. 2018ZX10734-404), the National Sharing Service Platform for Parasite Resources (Grant No. NPRC-2019-194-30).

Conflicts of Interest: The authors have declared that no competing interests exist.

The funders had no role in the design and implementation of the study, as well as other procedures related to preparation and submission of this article.

\section{doi: $10.46234 / \mathrm{ccdcw} 2020.176$}

\# Corresponding authors: Lin Ai, ailin@nipd.chinacdc.cn; Jiaxu Chen, chenjx@nipd.chinacdc.cn.

\footnotetext{
National Institute of Parasitic Diseases, Chinese Center for Disease Control and Prevention; Chinese Center for Tropical Diseases Research; Key Laboratory of Parasite and Vector Biology, Ministry of Health; National Center for International Research on Tropical Diseases, Ministry of Science and Technology; WHO Collaborating Center for Tropical Diseases, Shanghai, China; ${ }^{2}$ Shenzhen Center for Disease Control and Prevention, Shenzhen, China; ${ }^{3}$ National Institute of Parasitic Diseases, Chinese Center for Disease Control and Prevention-Shenzhen Center for Disease Control and Prevention Joint Laboratory for Imported Tropical Disease Control, Shanghai, China. ${ }^{\&}$ Joint first authors.
}

Submitted: July 13, 2020; Accepted: August 07, 2020

\section{REFERENCES}

1. Vannier E, Krause PJ. Human babesiosis. N Engl J Med 2012;
366(25):2397 - 407. http://dx.doi.org/10.1056/NEJMra1202018.

2. Ord RL, Lobo CA. Human babesiosis: pathogens, prevalence, diagnosis, and treatment. Curr Clin Microbiol Rep 2015;2(4):173 - 81 . http://dx.doi.org/10.1007/s40588-015-0025-z.

3. Barreira JD, Rossi MID, Pires FA, da Silva GVO, Massard CL. The effect of different strains Babesia bovis (Babes, 1888) on tick of Boophilus microplus (Canestrini, 1887). Rev Bras Parasitol Vet 2006; 15(4):138 - 42.

4. Skrabalo Z, Deanovic Z. Piroplasmosis in man; report of a case. Doc Med Geogr Trop 1957;9(1):11 - 6.

5. Krause PJ. Human babesiosis. Int J Parasitol 2019;49(2):165 - 74 . http://dx.doi.org/10.1016/j.ijpara.2018.11.007.

6. Gray EB, Herwaldt BL. Babesiosis surveillance-United States, 20112015. MMWR Surveill Summ 2019;68(6):1 - 11. http://dx.doi.org/ $10.15585 / \mathrm{mmwr} . s 56806 \mathrm{a} 1$.

7. Hildebrandt A, Gray JS, Hunfeld KP. Human babesiosis in Europe: what clinicians need to know. Infection 2013;41(6):1057-72. http://dx.doi.org/10.1007/s15010-013-0526-8.

8. Gorenflot A, Piette M. Seventh world case of human babesiosis (piroplasmosis). Scanning electron microscopic view of blood parasites. Ann Pharm Fr 1976;34(3 - 4): 89 - 94. (In French).

9. Symmers WS. Babesiosis in man. Br Med J 1979;2(6198):1141. http://dx.doi.org/10.1136/bmj.2.6198.1141-b.

10. Guirao-Arrabal E, González LM, García-Fogeda JL, Miralles-Adell C, Sánchez-Moreno G, Chueca N, et al. Imported babesiosis caused by Babesia microti-A case report. Ticks Tick Borne Dis 2020;11 (4):101435. http://dx.doi.org/10.1016/j.ttbdis.2020.101435.

11. Lim PL, Chavatte JM, Vasoo S, Yang J. Imported human babesiosis, Singapore, 2018. Emerg Infect Dis 2020;26(4):826-8. http://dx.doi.org/10.3201/eid2604.200025.

12. Hung SL. Notes on a species of malaria parasite finding from Bei-Bei. Nat Med J Chin (Chongqing ed) 1944;19:571 - 3. (In Chinese).

13. Jiang JF, Zheng YC, Jiang RR, Li H, Huo QB, Jiang BG, et al. Epidemiological, clinical, and laboratory characteristics of 48 cases of "Babesia venatorum" infection in China: a descriptive study. Lancet Infect Dis 2015;15(2):196 - 203. http://dx.doi.org/10.1016/s1473-3099 (14)71046-1.

14. Jia N, Zheng YC, Jiang JF, Jiang RR, Jiang BG, Wei R, et al. Human babesiosis caused by a Babesia crassa-like pathogen: a case series. Clin Infect Dis 2018;67(7):1110 - 9. http://dx.doi.org/10.1093/cid/ciy212.

15. Chen ZT, Li HQ, Gao XG, Bian AN, Yan HR, Kong DL, et al. Human babesiosis in China: a systematic review. Parasitol Res 2019; 118(4):1103 - 12. http://dx.doi.org/10.1007/s00436-019-06250-9.

16. Wang JM, Zhang SD, Yang JQ, Liu JL, Zhang DK, Li YQ, et al. Babesia divergens in human in Gansu province, China. Emerg Microbes Infect 2019;8(1):959 - 61. http://dx.doi.org/10.1080/22221751.2019. 1635431.

17. Li SH, Zhao YL, Gao LJ, Zhang YL, Zhou RM, Qian D, et al. Analysis of molecular epidemiology of babesiosis in patients having fever and thrombocytopenia in Xinyang City, Henan Province. Chin J Parasitol Parasit Dis 2019;37(1):66 - 9. http://dx.doi.org/10.12140/j.issn.10007423.2019.01.012. (In Chinese).

18. Wang H, Qi WJ. A case report of human Babesia infection in Beijing. Chin J Exp Clin Infect Dis (Electron Ed) 2019;13(6):519 - 23. http://dx.doi.org/10.3877/cma.j.issn.1674-1358.2019.06.012. (In Chinese).

19. Zhou X, Xia S, Huang JL, Tambo E, Zhuge HX, Zhou XN. Human babesiosis, an emerging tick - borne disease in the People's Republic of China. Parasit Vectors 2014;7:509. http://dx.doi.org/10.1186/s13071014-0509-3.

20. Man SQ, Qiao K, Cui J, Feng M, Fu YF, Cheng XJ. A case of human infection with a novel Babesia species in China. Infect Dis Poverty 2016;5:28. http://dx.doi.org/10.1186/s40249-016-0121-1.

21. Vannier EG, Diuk-Wasser MA, Ben Mamoun C, Krause PJ. Babesiosis. Infect Dis Clin North Am 2015;29(2):357 - 70. http://dx.doi.org/10. 1016/j.idc.2015.02.008. 